\title{
Les Pactes locaux : un processus médiateur pour une coopération territoriale
}

\author{
Pascal Mullard ${ }^{1}$ et Marie-France Dubois ${ }^{2}$ \\ Collectif national des Pactes locaux
}

\section{Qu'est ce qu'un Pacte local ?}

Le manifeste du collectif des Pactes locaux de septembre 1998 a été élaboré suite au constat du dysfonctionnement des différents dispositifs conçus et engagés par l'État pour lutter contre les exclusions et la pauvreté, et censés permettre de retrouver de la cohésion sociale. Le constat, aujourd'hui, est que manifestement la « mécanique » n'est pas adaptée...

Qu'est ce qu'un Pacte local pour nous? «Un Pacte local, c'est une dynamique collective territoriale, un processus construit dans la durée, permettant à tous les acteurs concernés, unis par des valeurs, de construire, de façon contractuelle, des réponses adaptées aux spécificités de chaque société locale, en particulier ses spécificités culturelles $»^{3}$.

\section{Le Pacte local répond à un besoin}

Les Pactes locaux répondent à un besoin à la fois très ancien et très actuel : c'est celui d'être en lien. Chacun, et quelle que soit sa place dans la société, est acteur parce qu'il est relié. Ce lien se construit en partie sur la base d'une appréciation personnelle du monde : qui est qui, qui fait quoi, et comment?

Cette appréciation est rendue difficile par la complexité de la société. Sur la même planète vivent et agissent des personnes et ensembles de personnes aux croyances différentes, implantées dans des réalités géographiques et climatiques différentes, succédant à des Histoires différentes. Ces personnes et ensembles de personnes auront donc des logiques différentes : un rapport au temps et à l'espace qui n'est pas le même, un rôle et un pouvoir qui n'ont pas le même poids.
Pour autant, le défi de base pour chacun est identique : satisfaire ses besoins (besoins physiologiques, psychologiques, spirituels), tout en prenant en compte « l'état de finitude» des ressources nécessaires à la satisfaction de ces besoins. Certaines ressources sont en effet infinies et d'autres non. En tout état de cause, elles sont, à la base, « à tout le monde ».

Sauf à penser le monde en termes de rapport de forces où le plus fort prend ce dont il a besoin sans s'inquiéter ni de la survie du plus faible ni de la reproduction des ressources, il y a donc une nécessité, pour les humains, de s'organiser, c'est-à-dire de penser les articulations entre les différentes nécessités et rationalités de chacun, entre les ressources et les contraintes. Ce défi planétaire, on le retrouve à tous les niveaux de l'échelle géographique : le continent, le pays, la région, le «bassin de vie».

\section{Le Pacte local est ancré sur le territoire}

On peut appeler « territoire » l'unité géographique à la fois bassin de vie et d'emploi, telle qu'essaient de les cerner les dispositifs de «Pays», de "Communauté de communes $» . .$. Comme on a pu le lire, on peut appeler ce territoire la «brique de base », l'acteur clé du développement de $21^{\mathrm{e}}$ siècle; on y retrouvera la complexité dont nous avons parlé. Des acteurs aux rationalités différentes œuvrant, chacun à leur place, pour relever le défi de base.

Le Pacte répond à ce besoin essentiel de coopérer pour vivre ensemble : c'est un processus qui rassemble des personnes qui, sur un territoire donné, ont des intérêts, des enjeux en commun et qui, par là même, s'engagent à partager une vision solidaire, humaniste, 
de la vie en société; et ce, notamment sur des thèmes comme l'insertion, l'environnement, le développement de l'emploi, la citoyenneté. Il n'est pas seulement un moyen de faire ensemble, une méthode, un outil. Il est un processus qui exprime des valeurs : la solidarité, et la convivialité ${ }^{4}$.

\section{Ce qui fonde le Pacte local... et sa fonction d'intermédiation}

Intermédiation que s'efforce d'abord d'exercer notre collectif lorsqu'il intervient sur un territoire donné, à la demande des acteurs locaux ou parce qu'il a repéré une action correspondant à ses critères et intéressante à promouvoir largement; car son rôle est de construire, faire connaître, mobiliser, apprécier, mettre en évidence et accompagner, à l'aide d'une grille de références à laquelle les acteurs de terrain et les cadres (financeurs, pouvoirs publics) peuvent s'adosser pour faire le point, développer, mettre en réseau, aider, financer, voire « modéliser ». Notre collectif sert donc d'intermédiateur entre les opérateurs eux-mêmes et avec les pouvoirs publics afin de faire avancer le développement du territoire.

Puis le Pacte en lui-même a une fonction d'intermédiation. En fait, il existe aujourd'hui de nombreux Pactes locaux qui ont des finalités et des modalités de fonctionnement différentes. Ils ont en commun ce que nous nommons les "fondamentaux»: ces éléments qui le constituent, et que l'on retrouve dans chacun des Pactes ${ }^{5}$, permettent l'expression concrète de valeurs telles que le fonctionnement démocratique, la solidarité, la convivialité.

Un Pacte local est tout d'abord "une démarche visant à associer : a minima les personnes concernées et deux autres catégories d'acteurs du territoire dont les élus, les institutions, le secteur économique (particulièrement PME et PMI), le secteur associatif, des personnes ressources, des compétences complémentaires et de façon optimale, avec le plus grand nombre ${ }^{6}$.

Du point de vue qui est le notre aujourd'hui, le Pacte est donc un espace de travail qui permet, par la mise en œuvre de ses «fondamentaux», aux différents acteurs de s'exprimer et de s'articuler. C'est le point de départ de sa «fonction d'intermédiation». Un des « fondamentaux » essentiels pour assurer la médiation est la présence du contrat : «Un PL existe à partir $d u$ moment où il y a concertation et engagement contractualisé. Le contrat peut revêtir plusieurs formes et contenir plusieurs éléments. Il semble néanmoins que cette contractualisation doive porter sur les objectifs, la mise en cuvre et les modalités d'évaluation; on parle, par exemple, de "diagnostic constamment réapprécié”».

La notion de contrat renvoie à celle d'engagement, mais aussi, au préalable, à celles de choix et de négociation. Le contrat est un espace de médiation en ceci que sa préparation est une période de travail qui permet à chacun de proposer ce qu'il souhaite apporter et retirer du Pacte, sur les conditions dans lesquelles il compte le faire. Le contrat garantit donc l'autonomie de chacun par rapport à l'autre. Comme le dit l'extrait, «le contrat peut revêtir différentes formes (statuts d'association, charte...)».

Un contrat est un outil de médiation; il est aussi le résultat d'un travail. Nous avons pensé que, pour que ce travail puisse se faire, il est nécessaire qu'au sein du Pacte, il y ait la «la présence de capacités à communiquer, à mettre en synergie des compétences, à mailler ». Si cette capacité peut s'incarner de différentes manières là encore (capacité collective, individuelle...), elle est en général apportée par les initiateurs ou animateurs du Pacte.

\section{Le contrat peut revêtir plusieurs formes et contenir plusieurs éléments.}

Le contrat s'appuie sur un «fondamental» complémentaire : c'est «l'identification claire des niveaux d'implication de chacun des acteurs ». En effet, une des conditions de la réussite de la négociation puis de la coopération est, dans un mouvement double :

1) que chacun ait soigneusement clarifié pour soi la position de laquelle il ou elle parle (dépasser les postures « multi-casquettes »);

2) que chacun ait la connaissance de la position de l'autre.

Cet élément fondamental peut paraître simpliste et évident; nous savons pourtant à quel point il est compliqué à atteindre. Les expériences de Pacte local sur lesquelles nous avons travaillé nous apprennent que cette identification est possible pour deux raisons : la 
première, c'est que les Pactes affichent un «esprit de recherche-action", ainsi qu' "une remise en question, (à travers des actions significatives) des systèmes relationnels, du fonctionnement des institutions, $d u$ sens commun (culture locale...), du consensus...». Ainsi, c'est le côté expérimental du Pacte, la cassure qu'il provoque dans les consensus affichés qui permettent de " prendre le risque » de fonctionner autrement. Ceci s'appuie certainement sur un diagnostic partagé des impasses locales liées aux manières de fonctionner des systèmes relationnels en place. La prise de conscience collective dont nous avons parlé plus haut a donc une première fonction : celle de faire partager la nécessité de travailler autrement. Le deuxième élément qui rend possible l'identification des niveaux d'implication des acteurs est toujours la présence d'une capacité à "prendre le risque de lancer la dynamique, prendre des initiatives, positiver les divergences pour qu'elles servent le bien commun, produire de l'estime mutuelle»; capacité qui peut émaner d'une seule personne et se propager à l'ensemble du collectif.

La convivialité et une certaine forme d'intimité entre les acteurs favorisent les échanges nécessaires à l'élaboration d'une interconnaissance et la naissance de la confiance.

\section{L'individuel et le collectif... qu'est-ce que produisent les Pactes ?}

Un résultat de l'intermédiation est ce que nous avons appelé «passer d'une prise de conscience individuelle à une prise de conscience collective ». Nous constatons que, partout où il y a Pacte, il y a une capacité (là encore bien souvent apportée par les initiateurs) à donner du sens à l'action collective. Ce sens est donné à partir de la connaissance, de la compréhension et de l'expression des réalités, ambitions et contraintes de chacun des acteurs du Pacte, d'une vision « ressource » qui, à partir du partage de compétences, fasse apparaître les interactions possibles et, enfin, d'une capacité à démontrer l'efficacité des attitudes coopératives. Ces capacités s'exercent autant dans l'animation du collectif que dans le contact individuel, qui favorise une expression différenciée, s'apparentant dans certains cas à de l'accompagnement vers une autre manière de faire. La convivialité et une certaine for- me d'intimité entre les acteurs favorisent les échanges nécessaires à l'élaboration d'une interconnaissance et la naissance de la confiance. On affirme ici la nécessité de la réussite affective interpersonnelle dans la mise en œuvre d'un processus de coopération territorial.

Ainsi, et pour être plus concret, certains Pactes ont permis la médiation par exemple entre des professionnels, des institutionnels, des bénévoles, et d'autres, entre des entreprises et des salariés, ou encore entre entreprises, élus et associations locales pour produire de l'emploi (sous forme de services aux entreprises ).

Le Pacte produit, naturellement, et en dehors du processus, ce pourquoi il est destiné : création d'activités, de services, négociations collectives... Il produit également, conformément à l'enjeu qui le définit, "plus de cohésion sociale, plus de solidarité, de participation démocratique et d'échanges entre les sphères économiques et sociales ».

Nous pensons qu'il produit, en outre :

- "plus de cohérence entre les acteurs, les actions et les échelons territoriaux»;

- un système de production collective efficient;

- une évolution personnelle pour ses acteurs;

- une amélioration dans leurs relations aux autres;

- une redéfinition des manières de faire telle que le retour en arrière est impossible : un changement de paradigme sur l'action territoriale.

Ce que nous savons de ce que produit le Pacte au niveau individuel a deux sources : la première est notre propre expérience, la deuxième, des éléments recueillis lors d'enquêtes de terrain.

Le «bien-être avec l'autre dans le travail », apport maintes fois cité, se construit à partir de différents éléments :

- une reconnaissance et une valorisation des apports de chacun;

- une amélioration de la capacité à répondre aux besoins;

- l'acquisition d'une vision plus globale de l'action en question sur son territoire. 


\section{Faire Pacte, une vision optimiste}

Ainsi, mettre en place un Pacte local requiert une confiance en soi et en l'autre sur sa capacité à être efficace, et à la hauteur des exigences du Pacte : contractualiser, évaluer, chercher, échanger, agir... sur toute la durée du Pacte ${ }^{7}$. Faire Pacte, c'est peut-être, simplement, se déclarer capables, collectivement, de s'organiser pour trouver des réponses aux besoins que l'on rencontre. C'est, ensuite, s'engager dans l'action. Les éléments d'un Pacte ne sont pas à proprement parler innovants ou complexes; mais ils sont «fondamentaux », nécessaires à la réussite de l'action. Ils permettent l'établissement d'une communication sereine entre les personnes, laquelle rend possible le travail.

Nous pensons, finalement, que les Pactes locaux qui existent aujourd'hui préfigurent un modèle de travail collectif pour la société actuelle et future. Un modèle exigeant, certes, mais aussi une réponse aux défis que nous apporte le monde complexe dans lequel nous vivons.

\section{Un exemple de Pacte local : le dialogue social territorial en Poitou-Charentes}

Cette action est particulièrement intéressante pour illustrer ce qu'on entend par Pacte local. En effet, à partir d'une opportunité, d'un « déclic initial », on est passé ici d'une gestion des ressources humaines traditionnellement verticale, tant à l'interne dans les entreprises qu'au niveau de la gestion de la précarité par les dispositifs publics, à un espace de gestion partenariale des ressources humaines à l'échelle du bassin d'emplois.

À l'origine de cette initiative, un «déclic initial » constitué à la fois :

- par le constat fait par les syndicats CFDT et CGC locaux qu'en France, une multitude d'acteurs interviennent sur un même territoire pour la gestion de l'emploi et de l'exclusion, mais sans articulation entre eux;

- par la nécessité pour la plus grande entreprise locale, le carrossier Heuliez, d'embaucher rapidement mais temporairement 1400 personnes pour répondre à une commande importante reçue de Citroën : conscient qu'il fallait, dès l'embauche, penser au reclassement de ces personnes après que la commande eut été honorée, Heuliez a accepté de jouer le jeu d'une gestion collective du problème.

Le cadre institutionnel de départ a été le comité de bassin d'emploi qui préexistait. À partir de celui-ci, les deux syndicats initiateurs et un groupe d'entreprises ont créé un centre d'orientation comme si le bassin d'emplois constituait globalement une grande entreprise dont les habitants étaient les salariés effectifs ou potentiels. Cette approche correspond à l'intuition que le territoire est un acteur social majeur en devenir et qu'il doit se doter d'outils opérationnels de gestion, notamment de ressources humaines.

La difficulté, c'est le temps nécessaire pour que le partenariat puisse se mettre réellement en place dans la durée; un an pour que les partenaires commencent à parler le même langage, du temps aussi pour « inventer » les termes de ce partenariat.

\section{Le territoire est un acteur social majeur en devenir.}

La première étape en a été une de diagnostic et de construction de compréhension mutuelle. Elle a été menée avec les deux syndicats, treize entreprises représentant 3000 salariés, l'ANPE et un cabinet extérieur. Cette étape de diagnostic a visé à apprécier de façon détaillée la ressource humaine du bassin d'emplois en analysant les compétences détenues aussi bien par les salariés que par les chômeurs. Elle a permis de renforcer les liens entre la ressource humaine et les besoins par des actions d'information, d'orientation et de formation.

En même temps se mettait en place une cellule de reclassement. Dans cette démarche, tous les acteurs ont trouvé leur avantage: les syndicats en devenant un acteur local écouté; les entreprises en développant des services communs permettant de répondre à leurs besoins alors qu'ils étaient incapables d'y répondre de façon isolée; l'État, car au lieu d'apporter des réponses comme il le fait traditionnellement, était en situation d'élaborer les questions elles-mêmes avec les autres partenaires; le Conseil régional, qui se voyait une nouvelle fonction; les branches professionnelles ellesmêmes, qui, pour une fois, arrivaient aussi à toucher 
le public des petites et moyennes entreprises. Dès l'origine, cette approche s'est voulue ouverte sur l'extérieur et s'est inscrite dans un projet européen...

Le but pratique était de permettre à chaque personne de la région - salariée, précaire ou exclue - d'établir son propre portefeuille de compétences et de le valoriser. En parallèle, un travail a été mené avec les offrants d'emplois pour les inciter à mieux décrire les compétences réellement requises que par le simple énoncé du métier.

En conclusion, on peut constater plusieurs impacts caractéristiques de cette initiative sur le territoire :

- Elle s'enracine dans une culture collective préalable et, en même temps, transforme cette culture.

- Les relations sociales peuvent être violentes, par exemple entre entreprises et salariés, mais quand il s'agit de la survie du territoire, tous sont capables de se mettre d'accord sur une plate-forme minimum.

- En même temps, ce type de démarche change les formes de relations entre les personnes et les institutions. Il n'est pas facile de mesurer ces changements, mais ils se manifestent néanmoins par des changements de pratiques. Par exemple, dans les petites entreprises locales, il n'y a plus de licenciement sans plan de reconversion. Et, critère d'évolution culturelle, quand dans cet espace partenarial les personnes changent, les nouvelles entrent dans le jeu et en respectent les règles,

- Même si les conditions d'émergence de ce mode de gestion de l'emploi au niveau local ont été particulières, une fois qu'un modèle de pratique entre les acteurs a été établi, il peut inspirer d'autres contextes : aujourd'hui, dans le Bassin de Thouars, 60 entreprises travaillent ensemble dans le même esprit; et, au niveau régional de Poitou Charentes, la démarche a fait des petits en créant un modèle de référence (création par exemple de 200 groupements d'employeurs ruraux employant 500 salariés).

- Ce dispositif de gestion territoriale des ressources humaines permet aussi de faire s'exprimer de nouvelles attitudes à l'égard du travail (par ex. : volonté des jeunes de gérer eux-mêmes leur parcours professionnel avec plus de souplesse).
- Grâce à la richesse des échanges avec d'autres projets européens et à l'ouverture que cela a généré, le Conseil régional a créé, dans le du contrat de plan État-Région une nouvelle structure, le CISTE, gérée paritairement par l'ensemble des syndicats des organisations patronales et par le monde associatif pour analyser les situations des ressources humaines sur le territoire, travailler à la transférabilité des expériences locales, impulser de nouvelles initiatives, offrir des boîtes à outil de l'innovation sociale en s'appuyant sur le travail des chercheurs et des administrations.

\section{Une fois qu'un modèle de pratique entre les acteurs a été établi, il peut inspirer d'autres contextes.}

On peut aussi tirer de cette expérience plusieurs enseignements :

- parce qu'il a vu l'intérêt de cette démarche, le sous préfet s'est engagé personnellement;

- dans les institutions publiques, tous les agents ont besoin d'être valorisés, sont en recherche de sens : si on les invite à partager un projet collectif, ils ne se comportent plus en financeurs au sens strict mais en partenaires;

- il est nécessaire d'organiser la visibilité de la démarche sous forme, par exemple, d'un événement public permettant à chacun de valoriser sa contribution;

- les élus locaux ont joué le jeu à Bressuire en raison des liens personnels existant entre tous les initiateurs;

- le dialogue créé sur un sujet suscite un espace partenarial qui permet de traiter de manière nouvelle d'autres sujets (par ex. ici les 35 heures);

- finalement, ce qui s'invente ici, avec la combinaison de l'échelle locale, régionale et européenne, correspond bien à l'idée de développement progressif des outils opérationnels de territoires constitués en acteur social émergent.

Cette expérience de Poitou Charentes contribue à créer un espace de formation à la coopération qui fait pacte, au sens propre du terme. 


\section{Notes et références}

1 Pascal Mullard est co-gérant d'Espace Développement qui est un cabinet coopératif d'accompagnement au changement basé à Dijon. Il est intervenant en organisation et membre du Collectif national des Pactes locaux.

2 Marie-France Dubois est sociologue de formation, membre du collectif national des Pactes locaux, chargée de mission Formation/Emploi, développement de projets territoriaux pour différents organismes publics et privés.

3 D. Gontier, membre du Comité de pilotage national, journée de lancement du 10 janvier 2001.
4 C'est aussi pour cela qu'il est naturellement ouvert sur cette complexité du monde dont nous avons parlé : parce qu'on ne peut penser la complexité sans reconnaître la valeur de l'autre.

5 Des réunions de travail - ouvertes et régulières - permettent d'avancer dans la connaissance de ces fondamentaux.

6 Tous les passages en italiques sont extraits d'un document de travail : « Ce qui fait Pacte».

7 Un Pacte a une durée, pour ce qu'on en pense aujourd'hui, plutôt longue et pas forcément définie au départ. 